\title{
El Retorno de la Desafiante Tradición Crítica Latinoamericana. Sustrato Teórico en la Construción de Modelos Alternativos de Desarrollo
}

\author{
Monika Meireles (*) \\ Eduardo Martínez-Ávila(*)
}

Resumen: El objetivo de este ensayo es rescatar y reinterpretar, de manera muy breve y en líneas generales, las principales aportaciones de la tradición crítica latinoamericana. De ese examen, sobresaltan las nociones de subdesarrollo y de dependencia, respectivamente, por caracterizar las relaciones desiguales de los países periféricos con los países hegemónicos, esto en el sentido de retomarlos como categorías de análisis básicas para la reflexión sobre las alternativas de desarrollo que se abrirían en la región con los llamados "gobiernos progresistas".

Palabras claves: Tradición crítica latinoamericana, Desarrollo, Subdesarrollo, CEPAL, Teoría Marxista de la Dependencia.

\begin{abstract}
The objective of this essay is to recuperate and reinterpret, in a brief fashion and in broad strokes, the principal contributions on the Latin American critical tradition. Notions of underdevelopment and dependence, used to characterize the unequal relationships between peripheral and hegemonic countries, will be employed as categories of basic analysis in the examination of development alternatives that may present themselves in the region with the so called "progressive governments".
\end{abstract}

Keywords: Latin American critical tradition, Development, Underdevelopment, ECLAC, Marxist Theory of Dependency.

(*) Profesora de asignatura de la Facultad de Economía de la Universidad Nacional Autónoma de México (UNAM). E-mail: $<$ meireles@usp.br>.

(**) Estudiante de la Facultad de Economía de la UNAM. E-mail:<eduardomtzavila@hotmail.com>. Recebido em 17.2.2011 e aceito em 14.6.2011. 


\section{INTRODUCCIÓN}

La teoría del desarrollo, sobre todo desde la visión de América Latina, viene nuevamente cobrando fuerza. Tras tres décadas de continuas crisis financieras, resultantes de la fragilidad instaurada por parte de las prácticas neoliberales impulsadas en esas latitudes, obviamente no se pudo asistir una trayectoria de desarrollo sostenido de la región. En la actual configuración del capitalismo, en la cual hay clara preponderancia de la acumulación financiera, los países latinoamericanos experimentaron una profunda transformación en su estructura económica: el aparato productivo armado como elemento fundamental de la estrategia de desarrollo hacía dentro, por la vía de la industrialización, fue desarticulado y asistimos a la reinstauración de un modelo de crecimiento pautado en las exportaciones de bienes primarios.

La deconstrucción del modelo pautado en el fortalecimiento del mercado interno trascurrió en dos etapas. Primeramente, por la mano de gobiernos de corte autoritario, y, posteriormente, por gobiernos que conformaron la llamada "democracia tutelada" o periodo de restablecimiento de "democracias formales" en la región. Tal transición se dio a partir de una serie ajustes estructurales de orientación ortodoxa - en consonancia con la oleada del neoliberalismo en el mundo - Tales imposiciones, estipuladas por el gobierno de Estados Unidos e instituciones como el Fondo Monetario Internacional (FMI), Banco Mundial (BM) y Organización Mundial del Comercio (OMC) agudizaron la relación de dependencia en la región: el capital financiero se convirtió en la nueva cara de este lazo subordinado. Ante tal panorama, dentro de la formación académica, la tradición crítica latinoamericana fue dejada al olvido; los paradigmas dominantes cuestionaron la universalidad de la visión latinoamericana sobre la temática del desarrollo, se le tachó de ser una teoría ambigua, de manifestar una postura simple y mecanicista, de ser un análisis parcial y periférico, cuyo interés estaría restricto al "caso latinoamericano", o aún más, limitado a la región en un momento histórico especifico ${ }^{(1)}$.

Actualmente, pasado más de una década de que se experimentara en América Latina lo que se conoció como "guiñada a la izquierda" (2), se hace necesario formular los primeros balances del significado y alcance histórico de esas experiencias y de su capacidad de impulsar un modelo de desarrollo distinto del primario exportador. La preocupación por intentar salir adelante con políticas públicas diferentes de aquellas de orientación fondomonetarista es un hecho evidente, sobre todo en estos países. Sin embargo, hasta el momento, la reiteración en la apuesta en un modelo que no tiene su centro dinámico en el mercado interno sigue siendo el talón de Aquiles en la trayectoria de muchas de estas economías ${ }^{(3)}$.

(1) Beigel (2006) nos habla de tres etapas de la tradición crítica en el pensamiento latinoamericano. La primera seria la "vida", o el momento de auge de esa producción teórica. El segundo momento se caracterizaría por la "muerte", o estancamiento en los debates de esa naturaleza, y seria coincidente con el periodo neoliberal en la región. Por fin, destaca lo que sería la "resurrección", o el resurgimiento tanto del interés por la producción de los autores clásicos como por actualizar sus aportaciones y así conformarse una nueva caja de herramientas teóricas para analizar la complejidad del desarrollo latinoamericano actual.

(2) Ese giro, en el escenario político de diversos países latinoamericanos, se inicia como respuesta a la insatisfacción social con los resultados de la implementación del modelo neoliberal. Lo que históricamente marca la ascensión de los "gobiernos progresistas" en la región es la elección y el inicio del gobierno de Hugo Chávez Frías en 1998. En el cono del subcontinente, la tendencia inaugurada en Venezuela ganó fuerza, tanto el "lulismo", en Brasil, como el "kirchnerismo", en Argentina, son ejemplos de eso.

(3) En ese sentido, hacemos eco a la percepción de que la apuesta en el modelo extractivista, con especial destaque para la producción minera en los países andinos, es extremadamente cuestionable, "no por falta de cultura productiva o simple 
En ese contexto, sigue el persistente desafío: ¿cómo lograr una teoría del desarrollo para la región de América Latina con claras y reales opciones de bienestar social? Esta pregunta era inherente a la preocupación teórica de los trabajos de la tradición crítica latinoamericana.

Ya a finales de los años cuarenta, tanto Prebisch y la corriente estructuralista de la Comisión Económica para la América Latina y el Caribe (CEPAL), así como la crítica hecha por parte de la llamada Teoría Marxista de la Dependencia (TMD) enriquecieron invaluablemente la tradición del pensamiento social latinoamericano sobre los problemas del desarrollo de la región. Entender que las aportaciones teórico-metodológicas que se llevaron a cabo en América Latina, con especial esplendor en las décadas de 1960-1970, representan claras contribuciones contrahegemónicas, capaces de cuestionar de manera crítica no solo el papel de la industrialización sino la viabilidad de las relaciones de producción capitalista como sistema capaz modificar nuestras estructuras subdesarrolladas, constituye una tarea fundamental, en el campo académico, para que se reaviva el debate público sobre alternativas de desarrollo ${ }^{(4)}$.

Así siendo, el objetivo de este ensayo es rescatar y reinterpretar, de manera muy breve y en líneas generales, las principales aportaciones de la tradición crítica latinoamericana elaboradas por la CEPAL y la TMD. De ese examen, sobresaltan las nociones de subdesarrollo y de dependencia, respectivamente, que caracterizan las relaciones desiguales de los países periféricos con los países hegemónicos, esto en el sentido de retomarlos como categorías de análisis básicas para la reflexión sobre los destinos del desarrollo de la región.

\section{Del debate académico latinoamericano en la POSguerra a la Superación DEL EUROCENTRISMO}

La Guerra Fría, como contexto histórico, marcaba una pugna político-económicocultural donde cualquier idea que sonara a socialismo era rechazada en los círculos no-comunistas. La Revolución Cubana, la elección de Allende y una serie de movimientos sociales masivos le dieron un carácter inédito a la región; estructuras cuyo desarrollo se encontraba condicionado y delimitado por el entorno externo e interno exigían un panorama diverso, en el cual el camino de reformismo dubitativo y reticente no era suficiente.

Ante tal panorama, se efectuaron análisis histórico-estructurales que desenmascararon las ventajas que el discurso capitalista alardeaba. La complejidad a nivel teórico ya era identificada, el desenvolvimiento histórico del sistema capitalista de producción generó estructuras desiguales, su expansión en el globo acarreó la existencia de zonas de pujante desarrollo y otras de negativamente destacado atraso. El desarrollo desigual y combinado que

demonización de la actividad, sino porque constituye una síntesis acabada del mal desarrollo. Pero además, dicha minería pone en jaque a la democracia, porque avanza sin consenso de las poblaciones, generando todo tipo de conflictos sociales, divisiones en la sociedad, y una espiral de criminalización de las resistencias que sin duda abre un nuevo y peligroso capítulo de violación de los derechos humanos" (SVAMPA, 2011).

(4) Complementando lo anterior, además de ser necesario construir perspectivas que recuperen críticamente las aportaciones que tanto la CEPAL como la TMD emitieron, es imprescindible que se las enriquezca, a partir de las complejidades que demarcan el inicio del siglo XXI, como son: el medio ambiente, la perspectiva de género, los movimientos locales, obreros, indígenas, anti-raciales, campesinos y de diversidad sexual. 
define esa forma de expansión, tiene vigencia hasta la actualidad y no podrá ser revertido por la dialéctica propia de dicho modo de producción. En definitiva: el subdesarrollo no se supera recorriendo una serie de etapas. Así, el único camino viable y posible es la construcción teórica y práctica que tome la edificación del socialismo como referente.

Entendiendo el periodo actual como un tiempo de transición, en lo cual la crisis asume múltiples facetas, también se observa la crisis de los paradigmas dominantes eurocéntricos tanto en la esfera económica, política, social como académica. Abogar por la recuperación del legado teórico latinoamericano que abordó de manera crítica, la problemática regional a partir de una visión propia, significa buscar caminos para una superación mayúscula y definitiva del subdesarrollo.

Parte sustancial del discurso dominante de los últimos treinta años ha sido la imposibilidad del estudio crítico de la sociedad capitalista. Los golpes militares en la región latinoamericana han propiciado un replanteamiento de las luchas internas, la transformación estructural fue desplazada por luchas en pro de la democracia.

La caída de la Unión Soviética y el supuesto fracaso de la "alternativa comunista" con el desmoronamiento del "socialismo real" propiciaron la completa implantación y aceptación del capitalismo a nivel mundial. El sistema hegemónico ganó aún más fuerza mediante la globalización; cualquier crítica a la estructura simplemente expresaba una visión obsoleta, dogmática, o simplemente cargada de una ideología ya demodé. Bajo la visión dominante, el capitalismo logró erguir y mantener en pié los pilares conceptuales del liberalismo político de finales del siglo XVIII, cuya expresión más fidedigna se materializa en la tríade: libertad, igualdad y democracia. Estos retumban y reverberan por todo el mundo como los sólidos fundamentos sobre los cuales reposa una sociedad más desarrollada y defensora de valores entendidos como universales.

El Consenso de Washington y el modelo de la economía estadounidense arraigaron, desde una perspectiva multidisciplinar, la falsa percepción de imposibilidad de pensar en alternativas estructurales. La dificultad de imaginar la instauración de una organización económica-social distinta, impone el supuesto a nivel social de que el capitalismo no tiene fin (SANTOS, 2010). Son inúmeras las voces conservadoras que actualmente siguen reafirmando ese sinsentido. El antídoto a ese discurso fatalista encuentra en, el legado teórico y práctico de la vieja tradición crítica latinoamericana, el fundamento para la creación de una epistemología alterna, que represente la lucha emancipadora orientada a inventar "otro lugar": una heteropía cuyos cimientos sean los principios de redistribución relacionado con el establecimiento de la equidad y el principio del reconocimiento vinculado con la aceptación de las diferencias, significados que implícitamente conllevan a la autorreflexión y la creación de nuevos manifiestos (SANTOS, 2009).

El marco teórico de referencia para la construcción de alternativas ante los desafíos que nos presenta el siglo XXI no puede ignorar o menospreciar las aportaciones que la CEPAL emitió para la región latinoamericana. Aunque la fehaciente creencia en la industrialización como mecanismo para el desarrollo de las economías periféricas (PREBISCH, 1996, [1949]) en la década de 1950, que de alguna forma intentó seguir el mismo camino que 
recorrieron los países desarrollados ${ }^{(5)}$, fue cuestionada ante los resultados que el modelo de desarrollo nacional engendró( ${ }^{(6)}$, el abordaje cepalino, sobretodo lo de su generación fundacional, trae consigo valiosísimos puntos a la agenda de construcción de una Latinoamérica distinta, como es el caso del método de análisis historio-estructural.

La concepción cepalina del subdesarrollo se fue modificando en las dos primeras décadas posteriores a la publicación "El desarrollo económico en la América Latina y algunos de sus principales problemas" en 1949, también conocido como el Manifiesto latinoamericano, tal cual apodado por Albert Hirschman. La idea de superar el subdesarrollo mediante el crecimiento económico, a través del proceso de industrialización, se contrarrestó conforme los indicadores productivos crecían sin modificar incisivamente la estructura económico-social de la región, los aportes teóricos tendían a descifrar que tanto el desarrollo como subdesarrollo eran dos partes de un mismo fenómeno, lo que implicaba la necesidad de un análisis más allá del económico.

Celso Furtado (1964, [1961].), a partir de un análisis histórico-estructural y claramente multidisciplinar, establece que el subdesarrollo es una etapa histórica autónoma, o sea, que no forma parte de un mismo camino que conllevaría necesariamente a un grado superior de desarrollo. El proceso en Europa Occidental desarrollado en las últimas décadas del siglo XVIII, modificó el carácter cualitativo de la economía mundial caracterizado por la desestructuración de la economía artesanal y la expansión territorial. Las periferias que lograsen industrializarse se depararían con una configuración del mercado mundial sustancialmente distinta de aquella encontrada por las naciones de "desarrollo clásico", y sustancialmente distinto sería la forma y los resultados de su proceso de industrialización.

Cardoso y Faletto (1983, [1969]) formulan un análisis que encadena a la sociología y el análisis político con el campo económico. Así, examinan las formas de interacción de los diversos grupos, fuerzas y clases sociales de cada nación, y la relación de éstos con los grupos externos, como parte de la pugna inherente por alcanzar las estructuras dominantes donde consolidan sus intereses y objetivos en el proceso político, mecanismo que define la integración dentro de las economías nacionales y su relación con el mercado internacional. De tal forma que esas relaciones de dominación condicionan el carácter avanzado o atrasado de una nación; analizando el proceso histórico del capitalismo, revelado por la expansión comercial e industrial, la periferia se vincula en un mercado mundial cuyos sistemas productivos son diferenciables cuantitativa y cualitativamente. Se destaca, así, cómo el carácter subdesarrollado de ciertos países se configura a través de la posición o función que desempeñen en la estructura global, a partir de las relaciones sociales subordinadas con el exterior. Lo anterior es generado por la falta de desarrollo del aparato productivo nacional y, simultáneamente, por el constante conflicto entre las fuerzas sociales internas.

Ante tal panorama, la intelectualidad de filiación marxista de la región realizó aportaciones que van más allá y que cuestionan duramente la estrategia desarrollista. Parte de

(5) Camino que Chang y Grabel (2006) demostraron ser difícilmente reproducidos sin una acción estatal decisiva, como se puede notar cuando los autores resaltan el empleo de políticas de protección a las importaciones y de fomento a las exportaciones por parte de Gran Bretaña en el siglo XVIII y el papel de los Estados Unidos como la economía más proteccionista del mundo desde mediados del siglo XIX hasta la Segunda Guerra Mundial.

(6) Los niveles aceleradísimos del crecimiento económico de los "milagros" que se multiplicaron en los países latinoamericanos que lograron industrializarse también generaron una desigualdad en la distribución del ingreso importante. 
sus postulados básicos era desenmascarar la noción dual, que entendía que en la región existían dos estructuras básicas distintas, que casi de manera autónoma se presentaban: las estructuras arcaicas y las modernas. Del estudio de la realidad latinoamericana, tal como lo cita Furtado, la condición teórica del subdesarrollo latinoamericano, es resultado de algo que extrapola la caracterización de una economía dualista. Entender que lo que caracteriza el subdesarrollo es "la formación de una estructura híbrida, en las que un núcleo capitalista pasaba a coexistir, pacíficamente, con una estructura arcaica” (1964, p. 235), lo convierte en un fenómeno social propio, cuya superación es algo más complejo que la simple adopción de los pasos del take off predicados por Rostow.

El argumento de que existirían, en América Latina, sociedades modernas, vinculadas con la urbanización, la industrialización, el desarrollo e identificadas con el "progreso de las relaciones capitalistas", y que esa fuerza motriz modernizaría las estructuras agrarias, familiares, más asemejadas a las "relaciones feudales de producción" es fácilmente falsificado por Rodolfo Stavenhagen (1973). El autor, además, cuestiona la supuesta independencia o existencia de dinámicas propias para cada una de esas formas organizativas. Pese a las diferencias cualitativas entre las estructuras del binomio atrasado/moderno que contempla la categoría sociedad dual, se resalta que ambos, como factor clave, son fruto de un único proceso histórico que desarrolló las diversas estructuras, conformando una sola estructura socio-económica subdesarrollada. Esa estructura nace de la interacción entre el polo moderno y el arcaico, y se caracteriza por reproducir, al interior del país, la heterogeneidad estructural y de subordinación que se observan en la relación centro-periferia a nivel internacional. Profundizando la cuestión, las relaciones feudales de producción observadas en ciertas regiones de América Latina, en función del ciclo y la etapa económico-histórica mundial, propiciaron la instauración de economías estancadas, subdesarrolladas, a cambio del florecimiento económico y social de regiones nacionales y extranjeras, que bajo el predominio de un colonialismo interno, "subdesarrollaron" en mayor medida las zonas tradicionales proveedoras de materias primas y mano de obra barata.

En cuanto a la categoría dependencia, Cardoso y Faletto (1983, [1969]) elaboraron un esbozo atribuyendo a la carencia de autonomía en las decisiones de producción y consumo, debido al rol en la dinámica de los países centrales, el fundamento básico sobre el que detona las relaciones de subdesarrollo y la dependencia. Theotonio dos Santos reelabora la noción bajo otra perspectiva, la dependencia "significa tomar el desarrollo como resultado de la formación, expansión y consolidación del sistema capitalista. Tal perspectiva implica la necesidad de integrar en una sola historia, la perspectiva de la expansión capitalista en los países hoy desarrollados y sus resultados por él afectados. Pero no como […] simples efectos del desarrollo capitalista sino como su parte integrante y dominante" (DOS SANTOS, 1978, p. 301).

La configuración histórica del sistema capitalista de producción genera una estructura en la que los países con desarrollo tecnológico autónomo, predominio comercial, de capitales, con fuerza político-social, son capaces de imponer condiciones de explotación y extracción de los excedentes a regiones cuyo desarrollo se encuentra subdesarrollado y condicionado. El entendimiento fundamental de la TMD es resaltar el aspecto condicionante que tiene la integración subordinada con las sociedades desarrolladas, o sea, que la influencia de la situación 
periférica de la región enmarca los límites de su trayectoria de desarrollo, sin embargo, esa sí tiene márgenes de maniobra de acuerdo con la configuración de la correlación de fuerzas entre las clases sociales a nivel nacional (DOS SANTOS, 1978). En una palabra, la situación de dependencia es una variable clave, pero no es el único determinante en el porvenir de las economías periféricas. Así, la riqueza de esta visión radica en que esta teoría no representa una afirmación de tintes mecanicistas, que la condición de país dependiente no sella definitivamente las posibilidades de cambios estructurales radicales de ese. De hecho, en el fulcro de esa corriente se encuentra la clara perspectiva y creencia en la capacidad de transformación y redireccionamiento de los rumbos de América Latina por los actores sociales de la misma. Bajo la inspiración de esta teoría, dos propuestas surgen en el horizonte de la región: intentar modificar la superestructura de la sociedad por la vía del reformismo radical, remarcando siempre los limitantes que imposibilitan que el desarrollo del capitalismo dependiente de Latinoamérica conlleve a los patrones de vida experimentados en las economías centrales; o, dejar a un lado de manera definitiva la opción capitalista y construir una alternativa basada en la construcción del socialismo.

Ruy Mauro Marini (1981, [1973]) afirma que el desarrollo de América Latina se vincula estrechamente con la dinámica del capital internacional, condicionándose la dependencia a partir de la división internacional del trabajo. En esa, el rol de las economías desarrolladas se liga con la producción de manufacturas de consumo, mientras que la exportación de materias primas se da por parte de América Latina. Así, la región garantizaba, en la etapa histórica de consolidación del capitalismo a nivel mundial, la producción de bienes agropecuarios a los países desarrollados mientras éstos promovían el desarrollo de sus industrias, "la participación de América Latina en el mercado mundial contribuirá a que el eje de la acumulación en la economía industrial se desplace de la producción de plusvalía absoluta a plusvalía relativa [...]. Sin embargo el desarrollo de la producción latino-americana $[\ldots .$.$] se dará fundamentalmente en base a una mayor explotación del trabajador$ (Marini, 1981, [1973], p. 23).

Considerándose que el desarrollo del sistema capitalista de producción en su acumulación del capital en escala ampliada genera mayor desarrollo de las fuerzas productivas materiales en detrimento de la fuerza de trabajo ocupada, lo que genera que el comportamiento de la composición orgánica de capital - la relación del capital variable y el capital constante - aumente considerablemente, la tasa de ganancia tiende a disminuir, la proporción de ganancia que ya se ha concretado en el mercado disminuye debido a que la plusvalía obtenida del obrero en el proceso de producción se divide entre el componente constante que aumenta proporcionalmente a un ritmo mayor y el componente variable que disminuye, relativamente, debido a que la fuerza de trabajo ha sido reemplazada por la adquisición de los factores objetivos de la producción. Sin embargo, existen elementos que contrarrestan la caída de la tasa de ganancia, medidas que permiten resarcir la vigencia de las relaciones de producción capitalista a partir de la manutención de su rentabilidad. En particular la desvalorización de la fuerza de trabajo por debajo de su valor y la elevación del grado de explotación del trabajo. Tales son exactamente los mecanismos que Marini (1981) señala como medidas que definen la superexplotación del trabajo en América Latina. La lógica que impera en los países subdesarrollados permite paliar las contradicciones propias del sistema capitalista 
como mecanismo que reproduce la acumulación de capital pero que, a la par, engendra el empobrecimiento sistemático de la clase trabajadora.

\section{Algunas conclusiones}

El fracaso de los resultados en las políticas trazadas por instituciones multilaterales en la región latinoamericana a nivel económico y social, conjuntamente con la maduración de las demandas de numerosos movimientos sociales, ha promovido el ascenso de gobiernos de corte progresista, que encarnan una configuración radicalmente distinta a la pactada por la propuesta neoliberal.

Ante la inestabilidad de la estructura capitalista actual a nivel mundial, marcada por las graves distorsiones económico-financieras de Estados Unidos y la Unión Europea y su proyección de estancamiento las próximas décadas, pareciera se inaugura una etapa de transición. El entorno global y la región latinoamericana se encuentran plagados de movimientos alternos, propuestas y desafíos, donde resulta necesario buscar elementos de análisis que sean capaces de abarcar la complejidad de la realidad actual y que brinden servicio a la elaboración de propuestas alternativas de desarrollo. Así, debatir las aportaciones teóricas que tanto de la CEPAL como la TMD elaboraron, enriqueciéndolas a partir de los nuevos retos que caracterizan la configuración contemporánea del capitalismo, es uno de los caminos que se revitalizan.

Subrayar que la condición de subdesarrollo y dependencia que envuelve a la región latinoamericana es un aspecto fundamental en la elaboración de trayectorias alternativas de desarrollo es fundamental para encerrarse de una buena vez la falsa promesa de que la reinstauración de las exportaciones de los bienes primarios serviría de eje en un modelo comprometido con la mejoría de las condiciones sociales en los países del subcontinente. Ese capítulo de la historia económica regional ya fue experimentado, más de una vez, y los resultados fueron nefastos. Tampoco hay que hacerse la apología de un modelo en los moldes intocados del que se experimentó en la etapa de industrialización por sustitución de importaciones. Por otra parte, es necesario reconocer - y celebrar - la fuerza de los movimientos sociales, movimientos indígenas, campesinos, feministas, ambientalistas, en pro de la diversidad sexual, afrodescendientes, que se oponen a la imposición de una única forma de vida válida inherente al pensamiento dominante; tales movimientos representan alternativas que conciben la realidad bajo parámetros totalmente distintos a los desarrollados por la ideología capitalista, que son vitales para contraponer la exposición de un fascismo societario derramado por la lógica del mercado (SANTOS, 2009).

Se requiere la búsqueda incesante por crear nuevas formas de organización social que contrarresten a la globalización hegemónica. Urge la reestructuración del propio pensamiento alterno, que tome en cuenta el legado teórico del marxismo, la tradición crítica latinoamericana, la experiencia comunista, la nueva izquierda y el legado práctico del socialismo cubano, los gobiernos plurinacionales y multiculturales de Bolivia y Ecuador, y la insurgencia de los pueblos indígenas en Ecuador y México, pero que, a la par, contemple la redefinición del sistema de conocimiento dominante para la formulación de un pensamiento 
crítico capaz de elaborar una construcción emancipadora donde "el respeto a la autonomía del otro es fundamental para el crecimiento de un nosotros de nivel superior" (GONZÁLEZ CASANOVA, 2009, p. 375).

\section{REFERENCIAS BIBLIOGRÁFICAS}

BEIGEL, F. Vida, muerte y resurrección de las teorías de la dependencia. En: Crítica y teoría en el pensamiento social latinoamericano. Buenos Aires: Clacso, 2006.

CARDOSO, Fernando H. y FALETTO, Enzo. Análisis integrado del desarrollo. En: Dependencia y desarrollo en América Latina. México: Siglo Veintiuno Editores, 1983, [1969].

CHANG, Ha-Joon; GRABEL, Ilene. Reivindicar el desarrollo. Un manual de política económica alternativa. Barcelona: Intermón Oxfam, 2006.

FURTADO, Celso. Elementos de una teoría del subdesarrollo. En: Desarrollo y subdesarrollo. Buenos Aires: Editorial Universitaria, EUDEBA, 1964, [1961].

GONZÁlEZ CASANOVA, Pablo. De la sociología del poder a la sociología de la explotación, pensar América Latina en el siglo XXI. México: CLACSO, 2009.

MARINI, Ruy Mauro. Dialéctica de la dependencia. México: Editorial Era, 1981, [1973].

PREBISCH, R. El desarrollo económico de América Latina y algunos de sus principales problemas. El trimestre económico, n. 249, México: Fondo de Cultura Económica, 1996, [1949].

SANTOS, Boaventura de Sousa. Una epistemología del Sur. México: Siglo XXI, CLACSO, 2009.

Refundación del estado en América Latina. Perspectivas desde una epistemología del sur.

México: Universidad de los Andes, Siglo XXI, Siglo del Hombre editores, 2010.

SANTOS, Theotonio dos. Imperialismo y dependencia. México: Editorial Era, 1978.

STAVENHAGEN, Rodolfo. Tres ensayos sobre América Latina. Barcelona: Anagrama, 1973.

SVAMPA, M. Minería, maldesarrollo y democracia. Rebelión, 9 ago. 2011. Disponible en: <http:// www.rebelion.org/noticia.php?id=133705> Consultado en: 9. ago. 2011. 